\title{
Nuclear Structure Studies with High-Precision Mass Measurements of Spontaneous Fission Fragments at the FRS Ion Catcher
}

\author{
A. Spătaru ${ }^{1,2,3}$, D. Amanbayev ${ }^{4}$, S. Ayet ${ }^{3}$, D.L. Balabanski ${ }^{1,2}$, \\ S. Beck ${ }^{3,4}$, J. Bergmann ${ }^{4}$, P. Constantin ${ }^{1}$, T. Dickel $^{3,4}$, \\ H. Geissel ${ }^{3,4}$, C. Hornung ${ }^{3}$, N. Kalantar-Nayestanaki ${ }^{5}$, \\ G. Kripko-Koncz ${ }^{4}$, I. Mardor ${ }^{6,7}$, A. Mollaebrahimi ${ }^{3,4,5}$, \\ W.R. Plaß ${ }^{3,4}$, C. Scheidenberger ${ }^{3,4,8}$, M. Wasserheß ${ }^{4}$, J. Zhao ${ }^{3}$ \\ ${ }^{1}$ Extreme Light Infrastructure - Nuclear Physics, Horia Hulubei National Insti- \\ tute for R\&D in Physics and Nuclear Engineering, Măgurele 077125, Romania \\ ${ }^{2}$ Doctoral School in Engineering and Applications of Lasers and Accelerators, \\ University Polytechnica of Bucharest, Romania \\ ${ }^{3}$ GSI Helmholtz Centre for Heavy Ion Research GmbH, Darmstadt, Germany \\ ${ }^{4}$ II. Physikalisches Institut, Justus-Liebig-Universität Gießen, Germany \\ ${ }^{5}$ ESRIG, University of Groningen, Groningen, The Netherlands \\ ${ }^{6}$ Tel Aviv University, 6997801 Tel Aviv, Israel \\ ${ }^{7}$ Soreq Nuclear Research Center, 81800 Yavne, Israel \\ ${ }^{8}$ Helmholtz Forschungsakademie Hessen für FAIR (HFHF), GSI Helmholtzzen- \\ trum für Schwerionenforschung, Campus Gießen, 35392 Gießen, Germany
}

Received 15 October 2021

doi: https://doi.org/10.55318/bgjp.2021.48.5-6.535

\begin{abstract}
Nuclear mass is a key property of atomic nuclei. The accurate determination of nuclear masses provides information for the nuclear shell structure and nuclear deformations. The FRS Ion Catcher experimental setup at GSI, Darmstadt was used to perform mass measurements of ${ }^{252} \mathrm{Cf}$ fragments using a multiple-reflection time-of-flight mass spectrometer. The reported results are compared to previous measurements and their implication in the estimation of two-neutron separation energy is discussed.
\end{abstract}

KEY WORDS: fission fragments, mass measurements, two-neutron separation energy.

\section{Introduction}

Nuclear masses provide important information to fields such as nuclear structure and nucleosynthesis, through their connection to the binding energy $B(Z, N)$, 


\section{A. Spataru et al.}

defined in equation 1, and the two-neutron separation energy $S_{2 n}(Z, N)$, equation 2,

$$
\begin{gathered}
B(Z, N)=\left[Z \cdot m_{p}+N \cdot m_{n}-M(Z, N)\right] \cdot c^{2}, \\
S_{2 n}(Z, N)=B(Z, N)-B(Z, N-2),
\end{gathered}
$$

here, $m_{p}$ is the proton mass, $m_{n}$ the neutron mass and $M(Z, N)$ the mass of a nucleus with $Z$ protons and $N$ neutrons.

Accurate determination of nuclear mass values is critical in validating the theoretical trends of $B(Z, N)$ and $S_{2 n}(Z, N)$. They can be determined indirectly, from measurements of the energy released in reactions or decays, or directly, using mass spectrometry. In recent years, the technological development of the mass spectrometers [1] leads to an improvement of measured uncertainties and revealed discrepancies [2] with respect to results of indirect measurements.

An experimental campaign for mass measurements using fragments produced in spontaneous fission of ${ }^{252} \mathrm{Cf}$ took place at the FRS Ion Catcher (FRS-IC) at GSI, Darmstadt. In the experiment, mass measurements of about 80 nuclei, located above the $Z=50$ closed shell and at $N=90$, were performed. This region corresponds to the transition between two nuclear shapes, phenomenon known as shape-phase transition, which has been observed for several isotopes chains [3], including the Pr chain. However, some of the mass values used in these observations are the result of the indirect measurements.

The present FRS-IC experiments were undertaked with the idea to expand the direct mass measurements in the $N=90$ shape-phase transition region and verify the previous indirect measurements. The results discussed in this paper are related to the mass measurements of ${ }^{152,153} \mathrm{Pr}$. The obtained mass values have been compared to previous direct measurements and the corresponding $S_{2 n}$ calculations were compared to the AME20 [4] mass evaluation.

\section{Experimental Setup}

The FRS-IC [5] is a setup consisting of a Cryogenic Stopping Cell (CSC) [6,7], a Radio-Frequency Quadrupole (RFQ) [8] transport beamline and a MultipleReflection Time-of-Flight Mass-Spectrometer (MR-ToF-MS) [9]. It can be used in experiments for mass measurements of nuclei emerging from the Fragment Separator (FRS) [10] when placed at the final focal plane, or as a stand-alone instrument that measures ions produced by sources mounted inside the CSC. In both cases, the ions-of-interest (IoI) are thermalized inside the gas-filled CSC and are then extracted and transported to the mass spectrometer. The MR-ToFMS performs highly-accurate mass measurements, with resolving power up to $1,000,000$ [11], by measuring the time-of-flight (ToF) of the IoI in a closed path. In this experiment, a broadband mode covering more than 20 mass units with a mass resolving power of 260,000 was used. 


\section{Results}

The measured masses of ${ }^{152,153} \mathrm{Pr}$ discussed in this paper were performed using a ${ }^{252} \mathrm{Cf}$ source of $37 \mathrm{kBq}$ activity, mounted inside the CSC. A drawing of the setup used is shown in Figure 1. The measured ToF data were converted to mass values using the ToF-to-mass equation in [1]. The final mass values of the IoI were determined using a Hyper-Exponentially Modified Gaussian function [12]. The data evaluation procedure is developed at the FRS-IC and described in details in Ref. [1].

The two masses were previously measured directly using a Penning trap, ${ }^{152} \mathrm{Pr}$ [2] and ${ }^{153} \operatorname{Pr}[2,13]$. The deviation between the previous measurements and the FRS-IC results, calculated as the difference between them, with their corresponding uncertanties, is shown in Figure 2. The uncertainties of the evaluated FRS-IC results are consisting of statistical, calibration and setup uncertainties (e.g. voltage delays). In the case of the cited values from Ref. [2], the systematic and statistical uncertainties are quadratically added for the final value, while in the case of the cited values from Ref. [13], only statistical uncertainty is considered. In the cases of ${ }^{152,153} \mathrm{Pr}$ the results are in agreement. The somewhat larger uncertainties of the FRS-IC results are partly due to the fact that the MR-ToF-MS was not tuned to the highest possible mass resolving power.

The $\mathrm{S}_{2 n}$ values, resulting from the measurements of ${ }^{152,153} \mathrm{Pr}$, were calculated using equation 2 and are shown in red in Figure 3 as a function of $N$. They are compared with the AME20 values for the Pr isotopes, where the direct measurements are indicated in black and the values obtained from at least one indirect measurement are in yellow. The new FRS-IC results follow the decreasing trend of the AME20 values.

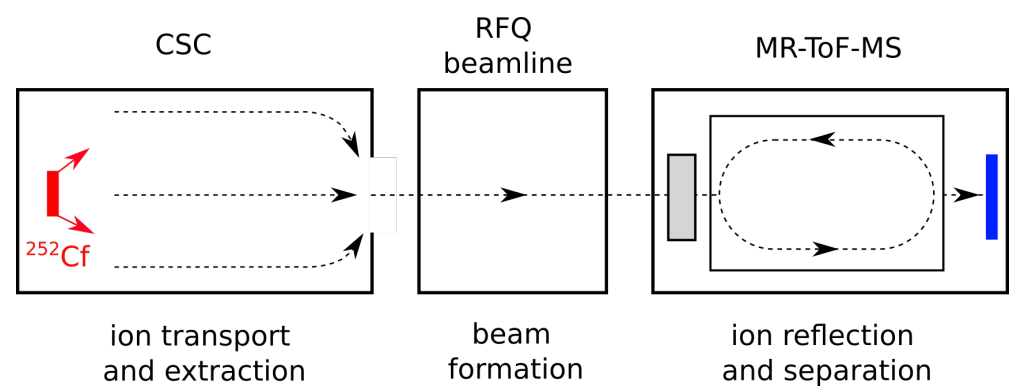

Figure 1. (Color online) Schematic representation of the FRS-IC setup used in the discussed measurements. The ${ }^{252} \mathrm{Cf}$ source, shown in red, was placed inside the CSC. The IoI are transported and extracted from the CSC and send to the MR-ToF-MS. There an injection trap, shown in gray, is storing the ions before they undergo several reflections inside an analyzer. In blue is the ToF detector at the end of the MR-ToF-MS. 
A. Spataru et al.

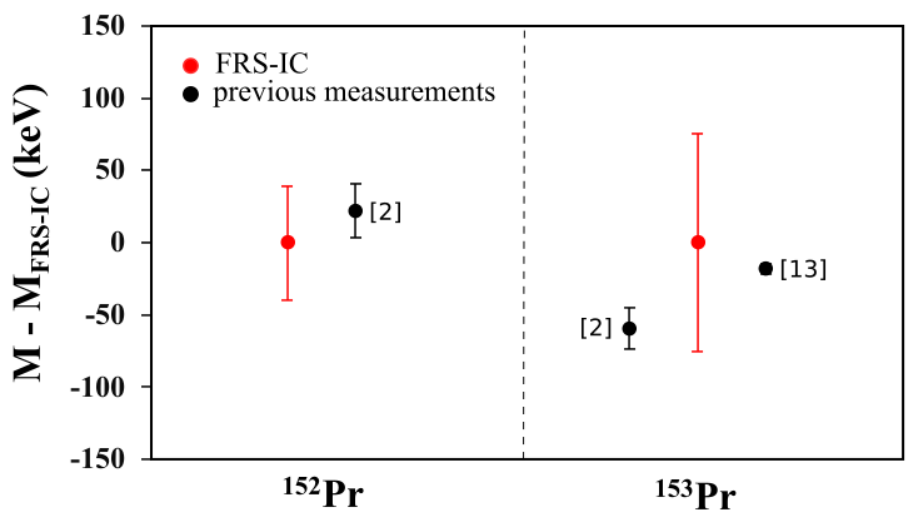

Figure 2. (Color online) Difference in keV between the measured masses with the FRSIC and previous direct measurements. The literature references of the previous measurements are indicated in paranthesis. Uncertainties are those of the specific measurement and not the propagated uncertainty of the difference.

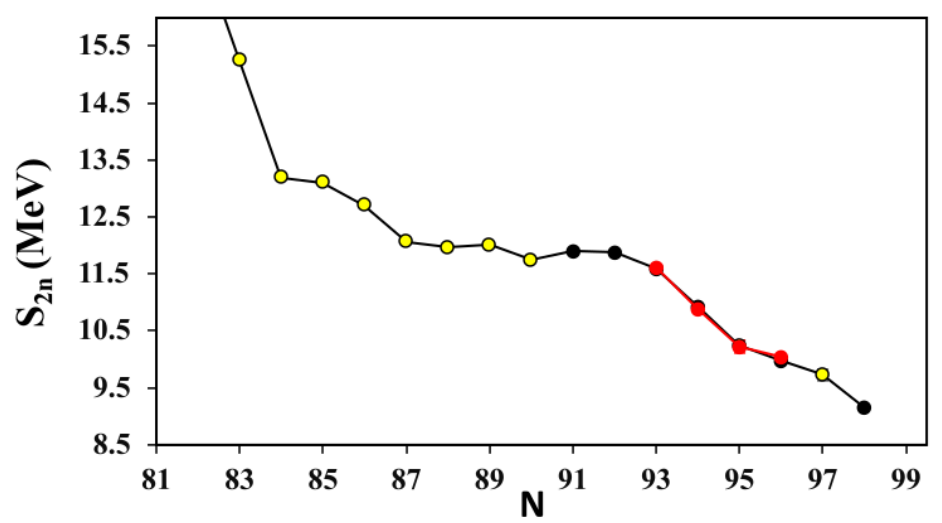

Figure 3. (Color online) Comparison between the two-neutron separation $\left(S_{2 n}\right)$ energies deduced from FRS-IC measurements (red) and values from the AME20 evaluation, direct measurements (black) and indirect estimates (yellow), for the case of Pr. Where not visible, the uncertainties are within the experimental points.

\section{Discussion and Conclusion}

The measurements of ${ }^{152,153} \mathrm{Pr}$, which were done with multiple-reflection timeof-flight mass spectrometry at the FRS-IC, show good agreement to the literature values $[2,13]$. This demonstrates the capabilities of the FRS-IC setup for direct mass measurements of spontaneous fission products. Direct mass measurements are crucial for the study of binding energy related observables, especially in 
cases when structural changes such as shape-phase transition are discussed. In such cases, the experimental evidence is the deviation of the $S_{2 n}$ values from a straight line, which can be influenced by systematic uncertainties from different measurements that are not taken into consideration.

\section{Acknowledgements}

The authors from ELI-NP acknowledge the support of the Romanian Ministry of Research and Innovation under research contract PN 19060105.

This work was supported by the German Federal Ministry for Education and Research (grant No. 05P19RGFN1, 05P21RGFN1), by the Hessian Ministry for Science and Art through the LOEWE Center HICforFAIR, by Justus Liebig University Gießen and GSI under the JLU-GSI strategic Helmholtz partnership agreement, and by HGS-HIRe

I.M. would like to acknowledge support by the Israel Ministry of Energy, Research Grant No. 220-11-052.

\section{References}

[1] S. Ayet San Andrés et al (2019) High-resolution, accurate multiple-reflection time-of-flight mass spectrometry for short-lived, exotic nuclei of a few events in their ground and low-lying isomeric states. Phys. Rev. C 99064313 DOI: https://doi.org/10.1103/PhysRevC.99.064313.

[2] G. Savard et al. (2006) Studies of neutron-rich isotopes with the CPT mass spectrometer and the CARIBU project. Int. J. Mass Spectrom. 251252 DOI: https://doi.org/10.1016/j.ijms.2006.01.047.

[3] D. Bucurescu and N.V. Zamfir (2017) Structure evolution and phase-transition in odd-mass nuclei. Phys. Rev. C 95014329 DOI: https://doi.org/10.1103/PhysRevC. 95.014329.

[4] M. Wang et al (2021) The AME 2020 atomic mass evaluation (II). Tables, graphs and references. Chinese Phys. C 45030003 DOI: https://doi.org/10.1088/16741137/abddaf.

[5] W. Plaß et al (2013) The FRS Ion Catcher - A facility for high-precision experiments with stopped projectile and fission fragments. Nucl. Instr. and Meth. in Phys. Res. B 317457 DOI: https://doi.org/10.1016/j.nimb.2013.07.063.

[6] M. Ranjan et al (2011) New stopping cell capabilities: RF carpet performance at high gas density and cryogenic operation. EPL 9652001 DOI: https://doi.org/10.1209/0295-5075/96/52001.

[7] S. Purushothaman et al (2013) First experimental results of a cryogenic stopping cell with short-lived, heavy uranium fragments produced at $1000 \mathrm{MeV} / \mathrm{u}$. EPL 104 42001 DOI: https://doi.org/10.1209/0295-5075/104/42001.

[8] M. P. Reiter et al (2011) Simulation der kryogenen Stoppzelle des FRS-IonCatchers-Experiments und Aufbau eines neuartigen FRQ-Strahlführungssystems. Master thesis, Justus-Liebig-University of Gießen 


\section{A. Spataru et al.}

[9] T. Dickel et al (2015) A high-performance multiple-reflection time-of-flight mass spectrometer and isobar separator for the research with exotic nuclei. Nucl. Instr. and Meth. in Phys. Res. A 777172 DOI: https://doi.org/10.1016/j.nima. 2014.12.094.

[10] H. Geissel et al (1992) The GSI projectile fragment separator (FRS): A versatile magnetic system for relativistic heavy ions. Nucl. Instr. and Meth. in Phys. Res. B 70286 DOI: https://doi.org/10.1016/0168-583X(92)95944-M.

[11] I. Mardor et al. (2021) Mass measurements of As, Se, and Br nuclei, and their implication on the proton-neutron interaction strength toward the $\mathrm{N}=\mathrm{Z}$ line. Phys. Rev. C 103034319 DOI: https://doi.org/10.1103/PhysRevC.103.034319.

[12] S. Purushothaman et al (2017) Hyper-EMG: A new probability distribution function composed of Exponentially Modified Gaussian distributions to analyze asymmetric peak shapes in high-resolution time-of-flight mass spectrometry. Int. J. Mass Spectrom. 421245 DOI: https://doi.org/10.1016/j.ijms.2017.07.014.

[13] J. Van Schelt et al. (2012) Mass measurements near the r-process path using the Canadian Penning Trap mass spectrometer. Phys. Rev. C 85252 DOI: https://doi.org/10.1103/ PhysRevC.85.045805. 